\title{
Value of Neoadjuvant Chemotherapy for Newly Diagnosed Advanced Ovarian Cancer: A European Perspective
}

\author{
Christina Fotopoulou, Imperial College London, London, United Kingdom \\ Jalid Sehouli, Charité Medical University of Berlin, Berlin, Germany \\ Giovanni Aletti, European Institute of Oncology, Milan, Italy \\ Philipp Harter, Kliniken Essen Mitte, Essen, Germany \\ Sven Mahner, Ludwig-Maximilians-University, Munich, Germany \\ Denis Querleu, Institut Bergonié, Bordeaux, France \\ Luis Chiva, Clínica Universidad de Navarra, Navarra, Spain \\ Hani Gabra, Imperial College London, London, United Kingdom \\ Nicoletta Colombo, European Institute of Oncology, Milan, Italy \\ Andreas du Bois, Kliniken Essen Mitte, Essen, Germany
}

The timing of debulking surgery in advanced ovarian cancer (AOC) has been the focus of debate and controversy in the international community for almost a decade. ${ }^{1-4}$ Although supporters of primary debulking surgery (PDS) advocate significantly better overall survival (OS) and progression-free survival rates, with even a significantly favorable impact on the patterns of relapse, ${ }^{4}$ opponents argue higher morbidity in a highly heterogeneous and often fatal disease. ${ }^{1-3}$ For more than two decades, we have known that each $10 \%$ increase in maximal cytoreduction is associated with a $5.5 \%$ increase in median survival, even after controlling for all other known patient- and tumor-specific variables. ${ }^{5,6}$ However, because of the unusual tumor biology and clinical behavior of AOC, with a typical diffuse peritoneal dissemination, in the majority of cases complete debulking is associated with multivisceral resections that require extensive surgical expertise, training, and infrastructural support to not lead to an exponential increase in morbidity. ${ }^{7}$

Neoadjuvant chemotherapy (NAC) and interval debulking surgery (IDS) have been considered as ways to reduce surgical morbidity; however, the oncologic safety of these approaches has never been proven in a maximal effort setting of high surgical expertise. ${ }^{2,3}$ The inconsistent quality of the surgical trials that have addressed this matter so far; the broad variation in practice nationally and internationally; and the still unanswered questions of fragility scores, biomarkers, and valid predictors of operability have led to strong polarization and controversy worldwide, which gives a clear signal of the need for further evidence.

As a network of European institutions dedicated to highquality gynecologic oncology care, we address some key points about this highly controversial topic and express some serious concerns related to a potential patient's compromise through suboptimal therapeutic strategies. Our statement is timed to the recent publication of the Society of Gynecologic Oncology and ASCO statement about the use of NAC in AOC, which provides guidance on the optimal timing of surgery and identifies diagnostic tools that facilitate the patient selection process. ${ }^{1}$

A multi-institutional study of 1,538 women with AOC treated at six National Cancer Institute-designated cancer centers showed that NAC is associated with shorter OS in stage IIIC disease compared with PDS (median OS, $33 v 43$ months; hazard ratio, 1.40; $95 \% \mathrm{CI}, 1.11$ to 1.77$){ }^{8}$ Although the study was not randomized, these findings add to the abundant clinical experience that patients who undergo PDS appear to have a better outcome in specialized, high-volume centers. ${ }^{6,-12}$

The two main phase III trials that have led to the broad implementation of NAC in AOC are the European Organisation for Research and Treatment of Cancer (EORTC) Neoadjuvant Chemotherapy or Primary Surgery in Stage IIIC or IV Cancer trial ${ }^{2}$ and Primary Chemotherapy Versus Primary Surgery for Newly Diagnosed Advanced Ovarian Cancer (CHORUS) trial. ${ }^{3}$ Both trials have generated findings that demonstrate a noninferior oncologic performance of NAC-IDS with lower surgical morbidity and mortality scores. On this basis, the Society of Gynecologic Oncology recommends that fit patients with seemingly resectable AOC receive either NAC-IDS or PDS as equivalent alternatives. ${ }^{1}$ How safe is it to extrapolate and project the findings of the EORTC and CHORUS trials to all patients with AOC, even though the patients and tumor profile represented in both studies show a clear selection bias as emphasized by the study authors. ${ }^{2,3}$ The low recruitment rate in CHORUS, with an average of only 1.35 surgeries per year in centers that serve $>1$ million patients, indicates a significant selection bias. A similar amount of selection bias is assumed in the EORTC trial in which large centers participated but recruited an average of 1.85 patients per year. In addition, the verylow complete resection rates in both trials suggest that mainly patients believed to be inoperable were recruited into both trials. 
The observation of poorer-than-expected patient characteristics fits well with the assumption that the tumor burden of the recruited patients was rather high, with $61.6 \%$ of patients in the EORTC trial having metastatic lesions $>10 \mathrm{~cm} .^{2}$ Furthermore, $20 \%$ of the patients in the CHORUS trial had a performance status of $\geq 2 .{ }^{3}$ The generalization of results from such highly selected adverse subgroups risks extrapolation to patients who are fit and present with potentially resectable disease. The extrapolation equivalence of PDS and NAC-IDS in this patient cohort is not justified and could lead to potentially curative treatment being withheld from a significant proportion of patients. We doubt that any chemotherapy trial would have received such broad recognition if only $20 \%$ of the standard chemotherapy dose were applied. Better survival rates after PDS in the subgroup of patients with metastases $<5 \mathrm{~cm}$ support this statement. ${ }^{13}$

The fact that the impact of residual disease becomes diluted in the NAC setting is a potential signal of chemotherapy-induced altered tumor biology. Despite a significant increase in complete tumor resection rates with IDS, this has failed to be translated into the expected equivalent increase in survival. ${ }^{2,3}$ This failure can only be explained by the hypothesis that completely different mechanisms apply in settings that have yet to be defined and may have to do with the effect of NAC selection, which may change the characteristics of the tumor encountered at NAC-IDS. ${ }^{14}$ Further trials that explore the mechanisms of platinum resistance induced by modality of treatment and that define the role of tumor biology in determining resectability and survival in relation to surgical effort are warranted.

The iatrogenic impact on quality of life (QoL) is another powerful tool of assessing treatment success. Physicians must share data with their patients about comparative morbidity, survival, and QoL in plain language to ensure that patients make more informed decisions. ${ }^{1}$ Opponents of radical PDS often argue that long-term impairment of postoperative QoL exists. In the EORTC and CHORUS prospectively randomized studies, no significant differences in QoL were identified between treatment arms; therefore, one cannot presume a long-term impairment in QoL derived from the higher radicality associated with PDS. ${ }^{2,3,15,16}$ Approximately $10 \%$ of patients did not undergo surgery in the neoadjuvant arms of either trial because of death, complications, or tumor progression before the scheduled IDS. Thus, only the fittest and most favorable patients were included in the postoperative adverse event analysis and interviewed about their postoperative QoL. This serious bias led to the decision to omit all statistical values for treatment burden analyses that presumably compared PDS and IDS in the EORTC trial publication. ${ }^{2}$

With all these challenges, patient selection is key to successful outcomes if we are careful not to expose patients unfit for surgery to radical cytoreduction and, on the other hand, not to withhold potential life-extending treatment from patients who would benefit. Even patients with stage IV disease have a significant survival benefit if operated tumor free compared with patients with unresectable disease for whom NAC or even primary chemotherapy should be considered. ${ }^{17}$ Preoperative examinations, such as with computed tomography (CT) imaging and CA-125 testing, have been described as valuable assessments of the extent of disease and the feasibility of surgical resection. ${ }^{1}$ Although a preoperative CT scan might detect inoperable metastases, it appears to have a low sensitivity for accurately describing tumor lesions at key sites. ${ }^{18}$ Numerous studies have shown that the prediction of suboptimal cytoreduction through CT imaging has significant limitations and cannot be cross validated. Therefore, CT imaging should not be used as a single tool to guide patient management. ${ }^{19-24}$ The same applies to testing CA125 levels, a specific cutoff of which has never been validated as a predictor of operability. We suggest that the limitations of all currently available tests to predict inoperability and overall surgical outcome be considered further. This area of research is ongoing, and until we have better tools, the clinical implementation of such methods should be done with caution because of their unproven reliability and risk to patients with resectable disease who might be denied surgery on the basis of unvalidated tools alone.

Diagnostic laparoscopy in a context of preoperative assessment has also been described as feasible and provides a reliable evaluation of the extent of disease burden. ${ }^{1}$ Laparoscopies may provide histologic tissue diagnosis and thus identify patients with low-grade histology who would not benefit from NAC for nonovarian neoplasms. However, their value in improving survival in unselected patient cohorts has never been shown. Laparoscopy can differentiate and select patients with low versus high tumor burden and result, as expected, in better survival and resectability figures for those with low tumor burden. ${ }^{25}$ This knowledge, though, does not help with the challenge of adequately treating patients with high tumor load who often are excluded from surgery by these laparoscopic series, which again leads to highly selected favorable results from patients with low tumor burden. ${ }^{26}$ So far, most series that used laparoscopy for selection have reported a higher rate of unsuccessful surgeries with residuals $>1 \mathrm{~cm}$ than in unselected series of consecutive patients with upfront debulking in expert centers. ${ }^{11,27,28}$ Novel strategies relying on predictive and prognostic biomarkers with innovative bioengineering tools, and radiomics classification features to define tumour dissemination patterns and biology in AOC may change the face of patient management and help to develop algorithms that will prospectively estimate the relative operative morbidity and benefit associated with PCS versus NAC and will objectify measuring residual disease. ${ }^{29}$

Surgical outcome is affected not only by tumor biology and patient-related factors that cannot be influenced, but also by surgical and infrastructural expertise. The European Society of Gynecologic Oncology recently published criteria for the quality of AOC surgery and recommended PDS in patients expected to undergo upfront debulking to no residual tumor with a reasonable (expected) complication rate. ${ }^{30,31}$ Caution should be heeded to not recruit NAC-IDS cases to fill the gaps that arise from suboptimal expertise and inadequate infrastructural setting.

We believe that surgical questions can only be answered sufficiently in studies with adequate surgical quality. This unmet need has resulted in the drive to design and now commence a further randomized trial to evaluate the optimal time point of surgery.

The Trial on Radical Upfront Surgery in Advanced Ovarian Cancer (TRUST), an initiative of the Arbeitsgemeinschaft Gynaekologische Onkologie study group together with the NorthEastern German Society of Gynaecologic Oncology, and international collaborators, has been designed to meet these criteria 
and has started recruitment. Participating centers went through a rigorous quality assessment protocol to confirm surgical and infrastructural quality. Quality assurance criteria for center eligibility include $\geq 36$ radical PDSs per year for stage IIIC and IV disease that achieve complete tumor clearance in $\geq 50 \%$ of cases. The criteria also include availability of the necessary infrastructure, such as an interdisciplinary setting, intensive care, and blood products. All centers were visited and audited at initiation, and regular central monitoring is performed. Furthermore, participating surgeons had to state their commitment to include and randomly assign all eligible patients to eliminate possible selection bias, such as referral of mainly fit patients in high-volume centers, and to record all patients with presumed AOC in a central registry. The complete abolishment of all possible clinical trial-related selection bias is impossible, but we have prospectively attempted to ameliorate and moderate these biases within TRUST through robust mechanisms of recruitment and documentation to better reflect clinical reality. Moreover, by accepting that high-volume centers may reflect a selection bias of patients who are able to reach these centers (ie, healthier, wealthier patients), we also equally accept that these centers function as tertiary referral destinations for cases deemed inoperable or more challenging for treatment in smaller centers. This means that patient cohorts in larger-volume centers often represent a collective of rather challenging, rare, and difficult cases that, again, may influence survival.

TRUST hopefully will not only further enlighten the adequate management of patients with AOC but also establish through the formation of a large translational platform predictive and prognostic biomarkers of operability and survival and identify valid fragility scores for the vulnerable population toward a more individualized surgical approach of this challenging disease.

\section{AUTHORS' DISCLOSURES OF POTENTIAL CONFLICTS OF INTEREST}

Disclosures provided by the authors are available with this article at ascopubs.org/journal/jco.

\section{AUTHOR CONTRIBUTIONS}

Manuscript writing: All authors

Final approval of manuscript: All authors

\section{REFERENCES}

1. Wright AA, Bohlke K, Armstrong DK, et al: Neoadjuvant chemotherapy for newly diagnosed, advanced ovarian cancer: Society of Gynecologic Oncology and American Society of Clinical Oncology clinical practice guideline. J Clin Oncol 34: 3460-3473, 2016

2. Vergote I, Tropé CG, Amant F, et al: Neoadjuvant chemotherapy or primary surgery in stage IIIC or IV ovarian cancer. N Engl J Med 363:943-953, 2010

3. Kehoe S, Hook J, Nankivell M, et al: Primary Chemotherapy Versus Primary Surgery for Newly Diagnosed Advanced Ovarian Cancer (CHORUS): An open-label, randomised, controlled, non-inferiority trial. Lancet 386:249-257, 2015

4. Petrillo $M$, Ferrandina $G$, Fagotti $A$, et al: Timing and pattern of recurrence in ovarian cancer patients with high tumor dissemination treated with primary debulking surgery versus neoadjuvant chemotherapy. Ann Surg Oncol 20: 3955-3960, 2013

5. Bristow RE, Tomacruz RS, Armstrong DK, et al: Survival effect of maximal cytoreductive surgery for advanced ovarian carcinoma during the platinum era: A meta-analysis. J Clin Oncol 20:1248-1259, 2002

6. du Bois A, Reuss A, Pujade-Lauraine E, et al: Role of surgical outcome as prognostic factor in advanced epithelial ovarian cancer: A combined exploratory analysis of 3 prospectively randomized phase 3 multicenter trials: by the Arbeitsgemeinschaft Gynaekologische Onkologie Studiengruppe Ovarialkarzinom (AGOOVAR) and the Groupe d'Investigateurs Nationaux Pour les Etudes des Cancers de I'Ovaire (GINECO). Cancer 115:1234-1244, 2009
7. Aletti GD, Dowdy SC, Gostout BS, et al: Quality improvement in the surgical approach to advanced ovarian cancer: The Mayo Clinic experience. J Am Coll Surg 208:614-620, 2009

8. Meyer LA, Cronin AM, Sun CC, et al: Use and effectiveness of neoadjuvant chemotherapy for treatment of ovarian cancer. J Clin Oncol 10.1200/ JCO.2016.68.1239 [epub ahead of print on September 6, 2016]

9. Chiva L, Lapuente F, Castellanos $T$, et al: What should we expect after a complete cytoreduction at the time of interval or primary debulking surgery in advanced ovarian cancer. Ann Surg Oncol 23:1666-1673, 2016

10. Sehouli J, Savvatis K, Braicu El, et al: Primary versus interval debulking surgery in advanced ovarian cancer: Results from a systematic single-center analysis. Int J Gynecol Cancer 20:1331-1340, 2010

11. Fotopoulou C, Jones BP, Savvatis K, et al: Maximal effort cytoreductive surgery for disseminated ovarian cancer in a UK setting: Challenges and possibilities. Arch Gynecol Obstet 294:607-614, 2016

12. Chang SJ, Bristow RE, Ryu HS: Impact of complete cytoreduction leaving no gross residual disease associated with radical cytoreductive surgical procedures on survival in advanced ovarian cancer. Ann Surg Oncol 19:4059-4067, 2012

13. van Meurs HS, Tajik $P$, Hof $M H$, et al: Which patients benefit most from primary surgery or neoadjuvant chemotherapy in stage IIIC or IV ovarian cancer? An exploratory analysis of the European Organisation for Research and Treatment of Cancer 55971 randomised trial. Eur J Cancer 49:3191-3201, 2013

14. du Bois A, Harter P. Understanding and optimizing the potential role of surgical debulking in advanced epithelial ovarian cancer. Am Soc Clin Oncol Ed Book e13-e17, 2010

15. Greimel E, Kristensen GB, van der Burg ME, et al: Quality of life of advanced ovarian cancer patients in the randomized phase III study comparing primary debulking surgery versus neo-adjuvant chemotherapy. Gynecol Oncol 131: 437-444, 2013

16. Leary A, Cowan R, Chi D, et al: Primary surgery or neoadjuvant chemotherapy in advanced ovarian cancer: The debate continues... Am Soc Clin Oncol Educ Book 35:153-162, 2016

17. Ataseven B, Chiva LM, Harter $P$, et al: FIGO stage IV epithelial ovarian, fallopian tube and peritoneal cancer revisited. Gynecol Oncol 142:597-607, 2016

18. Nasser $S$, Lazaridis $A$, Evangelou $M$, et al: Correlation of pre-operative CT findings with surgical \& histological tumor dissemination patterns at cytoreduction for primary advanced and relapsed epithelial ovarian cancer: A retrospective evaluation. Gynecol Oncol. 2016 143:264-269, 2016

19. Dowdy SC, Mullany SA, Brandt KR, et al: The utility of computed tomography scans in predicting suboptimal cytoreductive surgery in women with advanced ovarian carcinoma. Cancer 101:346-352, 2004

20. Nelson BE, Rosenfield AT, Schwartz PE: Preoperative abdominopelvic computed tomographic prediction of optimal cytoreduction in epithelial ovarian carcinoma. J Clin Oncol 11:166-172, 1993

21. Bristow RE, Duska LR, Lambrou NC, et al: A model for predicting surgical outcome in patients with advanced ovarian carcinoma using computed tomography. Cancer 89:1532-1540, 2000

22. Axtell $A E$, Lee $M H$, Bristow RE, et al: Multi-institutional reciprocal validation study of computed tomography predictors of suboptimal primary cytoreduction in patients with advanced ovarian cancer. J Clin Oncol 25: 384-389, 2007

23. Rutten MJ, van de Vrie R, Bruining $A$, et al: Predicting surgical outcome in patients with International Federation of Gynecology and Obstetrics stage III or IV ovarian cancer using computed tomography: A systematic review of prediction models. Int J Gynecol Cancer 25:407-415, 2015

24. Kim HJ, Choi $\mathrm{CH}$, Lee $Y Y$, et al: Surgical outcome prediction in patients with advanced ovarian cancer using computed tomography scans and intraoperative findings. Taiwan J Obstet Gynecol 53:343-347, 2014

25. Horowitz NS, Miller A, Rungruang $B$, et al: Does aggressive surgery improve outcomes? Interaction between preoperative disease burden and complex surgery in patients with advanced-stage ovarian cancer: An analysis of GOG 182. J Clin Oncol 33:937-943, 2015

26. Gómez-Hidalgo NR, Martinez-Cannon BA, Nick AM, et al: Predictors of optimal cytoreduction in patients with newly diagnosed advanced-stage epithelial ovarian cancer: Time to incorporate laparoscopic assessment into the standard of care. Gynecol Oncol 137:553-558, 2015

27. Heitz F, Harter P, Alesina PF, et al: Pattern of and reason for postoperative residual disease in patients with advanced ovarian cancer following upfront radical debulking surgery. Gynecol Oncol 141:264-270, 2016

28. Harter $P$, Muallem ZM, Buhrmann $C$, et al: Impact of a structured quality management program on surgical outcome in primary advanced ovarian cancer. Gynecol Oncol 121:615-619, 2011 
29. Bowtell DD, Böhm S, Ahmed AA, et al: Rethinking ovarian cancer II: Reducing mortality from high-grade serous ovarian cancer. Nat Rev Cancer 15 : 668-679, 2015

30. Querleu D, Planchamp F, Chiva L, et al: European Society of Gynaecologic Oncology quality indicators for advanced ovarian cancer surgery. Int J Gynecol Cancer 26:1354-1363, 2016
31. European Society of Gynaecologic Oncology: Recommendations for advanced ovarian cancer surgery. https://guidelines.esgo.org/ovarian-cancer/guidelines/advancedstage-surgery

DOI: 10.1200/JCO.2016.71.0723; published at ascopubs.org/journal/jco on January 9, 2017.

\section{Conquer Cancer Foundation of the American Society of Clinical Oncology}

The Conquer Cancer Foundation (CCF), created by the world's foremost cancer physicians of the American Society of Clinical Oncology, seeks dramatic advances in the prevention, treatment, and cures of all types of cancer. Working toward the vision of a world free from the fear of cancer, CCF invests in doctors and scientists at every stage of their career by funding breakthrough cancer research, sharing cutting-edge knowledge, and improving the quality of and access to care. For more information, visit CONQUER.ORG.

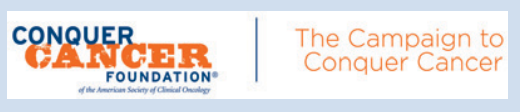




\section{AUTHORS' DISCLOSURES OF POTENTIAL CONFLICTS OF INTEREST}

Value of Neoadjuvant Chemotherapy for Newly Diagnosed Advanced Ovarian Cancer: A European Perspective

The following represents disclosure information provided by authors of this manuscript. All relationships are considered compensated. Relationships are self-held unless noted. I = Immediate Family Member, Inst = My Institution. Relationships may not relate to the subject matter of this manuscript. For more information about ASCO's conflict of interest policy, please refer to www.asco.org/rwc or ascopubs.org/jco/site/ifc.

\section{Christina Fotopoulou}

Consulting or Advisory Role: Roche, Tesaro, PharmaMar, AstraZeneca, Pharmacokinesis

\section{Jalid Sehouli}

Consulting or Advisory Role: Tesaro, Roche Pharma AG, Clovis

Oncology, AstraZeneca, Novocure

Research Funding: AstraZeneca (Inst), Eli Lilly (Inst)

\section{Giovanni Aletti}

No relationship to disclose

\section{Philipp Harter}

Honoraria: AstraZeneca, Roche

Consulting or Advisory Role: AstraZeneca, Roche, PharmaMar, Tesaro, Clovis Oncology

Travel, Accommodations, Expenses: Medac Pharma

\section{Sven Mahner}

Honoraria: Tesaro, AstraZeneca, Clovis Oncology, Medac Pharma, MSD, Roche, TEVA Pharmaceuticals Industries

Consulting or Advisory Role: Tesaro, AstraZeneca, Clovis Oncology, GlaxoSmithKline, Janssen-Cilag, Medac Pharma, MSD, PharmaMar, Roche, Sensor-Kinesis, TEVA Pharmaceuticals Industries Research Funding: Tesaro (Inst), AstraZeneca (Inst), GlaxoSmithKline (Inst), Janssen-Cilag (Inst), Medac Pharma (Inst), PharmaMar (Inst), Roche (Inst)

\section{Denis Querleu}

Consulting or Advisory Role: Roche

\section{Luis Chiva}

Honoraria: Roche, Takeda Pharmaceuticals, GlaxoSmithKline

Consulting or Advisory Role: Roche, AstraZeneca, Takeda

Pharmaceuticals

Speakers' Bureau: Roche, Takeda Pharmaceuticals, GlaxoSmithKline Travel, Accommodations, Expenses: Takeda Pharmaceuticals, Roche

\section{Hani Gabra}

No relationship to disclose

Nicoletta Colombo

Consulting or Advisory Role: Roche, Genentech, PharmaMar, Amgen, AstraZeneca, Clovis Oncology, Pfizer, MSD

Andreas du Bois

Consulting or Advisory Role: AstraZeneca, PharmaMar, Roche, Genentech, Mundipharma, Pfizer 